\title{
Erratum to: Senile Atopic Dermatitis
}

\author{
Ryoji Tanei
}

\section{Erratum to:}

Chapter 18 in: Ichiro Katayama/ Hiroyuki Murota / Takahiro Satoh (eds), Evolution of Atopic Dermatitis in the 21st Century

https://doi.org/10.1007/978-981-10-5541-6_18

The correction in page-239 was inadvertently missed and published. This has now been updated. The first reference citation in "18.2.6 Epidemiology" section was published incorrectly. This has also been updated now.

The updated online version of the original chapter can be found at https://doi.org/ 10.1007/978-981-10-5541-6_18

R. Tanei, M.D., Ph.D.

Department of Dermatology, Tokyo Metropolitan Geriatric Hospital and Institute of Gerontology, 35-2 Sakaecho, Itabashi, Tokyo 173-0015, Japan

e-mail: rtanei@aol.com; rtanei@tmghig.jp 